PROCEEDINGS OF THE

AMERICAN MATHEMATICAL SOCIETY

Volume 134, Number 5, Pages 1339-1345

S 0002-9939(05)08078-0

Article electronically published on August 29, 2005

\title{
ON BANACH SPACES WITH FEW SPREADING MODELS
}

\author{
BÜNYAMIN SARI
}

(Communicated by N. Tomczak-Jaegermann)

\begin{abstract}
If the set of spreading models of a Banach space $X$ is countable (up to equivalence), then it cannot contain a strictly increasing infinite chain of spreading models generated by normalized weakly null sequences. Moreover, such a space $X$ must have a spreading model which is 'close' to $c_{0}$ or $\ell_{p}$ for some $1 \leq p<\infty$.
\end{abstract}

\section{INTRODUCTION}

In this note we address a problem on the structure of the set of spreading models of Banach spaces, and make a few remarks on other related problems.

Recall that, by Ramsey theory, for every normalized basic sequence $\left(y_{i}\right)$ in a Banach space $X$ and for every $\left(\varepsilon_{n}\right) \searrow 0$ there exists a subsequence $\left(x_{i}\right)$ of $\left(y_{i}\right)$ and a normalized basic sequence $\left(\tilde{x}_{i}\right)$ in some Banach space $\tilde{X}$ such that: For all $n \in \mathbb{N}$, $\left(a_{i}\right)_{i=1}^{n} \in[-1,1]^{n}$ and $n \leq k_{1}<\ldots<k_{n}$,

$$
\left|\left\|\sum_{i=1}^{n} a_{i} x_{k_{i}}\right\|-\left\|\sum_{i=1}^{n} a_{i} \tilde{x}_{i}\right\|\right|<\varepsilon_{n} .
$$

The sequence $\left(\tilde{x}_{i}\right)$ is called the spreading model of $\left(x_{i}\right)$ and it is a suppression 1unconditional basic sequence if $\left(y_{i}\right)$ is weakly null. (See [BL] for further background on spreading models.)

Consider the set $S P(X)$ of all spreading models $\left(\tilde{x}_{i}\right)$ of a Banach space $X$ equipped with the partial order which is defined by domination. $\left(\tilde{x}_{i}\right) \geq\left(\tilde{y}_{i}\right)$ if for some $K<\infty, K\left\|\sum_{i} a_{i} \tilde{x}_{i}\right\| \geq\left\|\sum_{i} a_{i} \tilde{y}_{i}\right\|$ for all scalars $\left(a_{i}\right)$. We identify $\left(\tilde{x}_{i}\right)$ and $\left(\tilde{y}_{i}\right)$ in $S P(X)$ if $\left(\tilde{x}_{i}\right) \geq\left(\tilde{y}_{i}\right)$ and $\left(\tilde{y}_{i}\right) \geq\left(\tilde{x}_{i}\right)$. By $S P_{\omega}(X)$ denote the subset of $S P(X)$ which consists of spreading models generated by normalized weakly null sequences. A study of the structure of $S P(X)$ was initiated by Androulakis, Odell, Schlumprecht and Tomczak-Jaegermann AOST. In particular, they proved that every countable subset of $S P_{\omega}(X)$ admits an upper bound in $S P_{\omega}(X)$ (see Theorem 1.1 below).

In this note we consider a problem in this context posed to us by E. Odell. Suppose that $S P_{\omega}(X)$ contains a strictly increasing sequence $\left(\tilde{x}_{i}^{1}\right)_{i}<\left(\tilde{x}_{i}^{2}\right)_{i}<\ldots<$ $\left(\tilde{x}_{i}^{n}\right)_{i}<\ldots$ of spreading models. Does it follow that $S P_{\omega}(X)$ also contains an uncountable increasing chain of spreading models? We show that this problem has a positive answer (Theorem 2.2).

Received by the editors November 2, 2004 and, in revised form, November 23, 2004.

2000 Mathematics Subject Classification. Primary 46B20; Secondary 46B15.

(C)2005 American Mathematical Society Reverts to public domain 28 years from publication 
Examples of Banach spaces $X$ for which $S P_{\omega}(X)$ is infinite and does not contain an infinite increasing chain exist. For instance, if $2<p_{1}<p_{2}<\ldots$, then the space $X=\left(\sum \oplus \ell_{p_{i}}\right)_{\ell_{2}}$ is such an example. Clearly (the unit vector bases of), $\ell_{2}, \ell_{p_{1}}, \ell_{p_{2}}, \ldots$ form a decreasing infinite chain in $S P_{\omega}(X)$. Moreover, it is not difficult to show that, up to equivalence, these are the only spreading models of $X$ (cf. p. 20 of [AOST]).

It is an interesting open question whether a Banach space $X$ with few spreading models (e.g., finite or countable) must admit a $c_{0}$ or $\ell_{p}$ spreading model. This is not known even when $X$ has, up to equivalence, a unique spreading model. This case, a problem due to S. Argyros, is closely related to a problem raised by H. Rosenthal. If a Banach space $X$ has a basis $\left(e_{i}\right)$ with the property that every normalized block sequence has a subsequence which is equivalent to $\left(e_{i}\right)$, must $\left(e_{i}\right)$ be equivalent to the unit vector basis of $\ell_{p}$ or $c_{0}$ ? Exploiting this connection and using Theorem 1.1. some partial results to Rosenthal's problem were obtained in FPR. In the second part of the paper we obtain some improvements to these results. In particular, we show that if $X$ has countably many spreading models, then one of them must be 'close' to some $\ell_{p}$ or $c_{0}$ (see Theorem 3.4).

Throughout the paper we adapt the notation from [AOST. Our main tool is the following the upper bound theorem from [AOST, which we state in a slightly different (but equivalent) formulation.

Theorem 1.1 ([AOST] $)$. Let $\left(C_{n}\right) \subset(0, \infty)$ such that $\sum_{n} C_{n}^{-1}<\infty$ and let $\left(\tilde{x}_{i}^{(n)}\right)$, $n=1,2, \ldots$, be in $S P_{\omega}(X)$. Then there exists $\left(\tilde{y}_{i}\right)$ in $S P_{\omega}(X)$ and a constant $K$, where $K \leq\left(\sum_{n} C_{n}^{-1}\right)$, such that $\left(\tilde{y}_{i}\right) K C_{n}$-dominates $\left(\tilde{x}_{i}^{n}\right)$ for all $n \in \mathbb{N}$.

The value of the constant $K$ above, which is not stated in the original statement in AOST, can be easily verified by doing the proof carefully to obtain the best possible estimates. Moreover, it also follows from the construction in the proof of the theorem that there exists a constant $D \leq \min \left\{C_{n}: n \in \mathbb{N}\right\}$ such that for all $\left(a_{j}\right)_{j=1}^{m}$,

$$
\left\|\sum_{j=1}^{m} a_{j} \tilde{y}_{j}\right\| \leq D \sum_{i=1}^{\infty} C_{i}^{-1}\left\|\sum_{j=1}^{m} a_{j} \tilde{x}_{j}^{(i)}\right\| .
$$

The construction also yields that if the theorem is applied to finitely many $\left(\tilde{x}_{i}^{(n)}\right)$ for $n \leq m$, then (see remark 3.5(b) of [AOST] $)\left(\tilde{y}_{i}\right)$ in the theorem is equivalent to the norm given by

$$
\left\|\left(a_{i}\right)\right\|=\max _{n \leq m}\left\|\sum_{i} a_{i} \tilde{x}_{i}^{(n)}\right\| .
$$

In particular, if $\left(\tilde{x}_{i}^{(1)}\right)_{i} \leq\left(\tilde{x}_{i}^{(2)}\right)_{i} \leq \ldots \leq\left(\tilde{x}_{i}^{(m)}\right)_{i}$, then for all $m \in \mathbb{N},\left(\tilde{y}_{i}\right)_{i}$ above is equivalent to $\left(\tilde{x}_{i}^{(m)}\right)$.

\section{InCREASING CHAIN IMPLIES UNCOUNTABLE}

In the proof of the main theorem we will make use of the following transfinite induction principle (cf. Proposition 3.7 of [AOST]).

Lemma 2.1. Let $(M, \leq)$ be a non-empty partially ordered set satisfying the following two conditions:

(i) $M$ does not have a maximal element with respect to $\leq$. 
(ii) For every $\left(A_{i}\right) \subset M$ there exists $A \in M$ such that $A_{i} \leq A$ for every $i \in \mathbb{N}$.

Then for all ordinals $\alpha<\omega_{1}$ there exists $A^{\alpha} \in M$ such that if $\alpha<\beta<\omega_{1}$, then $A^{\alpha}<A^{\beta}$.

Theorem 2.2. Suppose that a Banach space $X$ admits a strictly increasing infinite sequence of spreading models generated by normalized weakly null sequences. Then $X$ also admits an uncountable strictly increasing chain of such spreading models.

It was shown in FPR (Proposition 9) that for a separable space $X$, the cardinality of $S P(X)$ is, up to equivalence, either continuum or at most countable. Thus we have

Corollary 2.3. If a separable space $X$ satisfies the assumption of the above theorem, then $X$ has continuum many non-equivalent spreading models.

Proof of Theorem 2.2. Let $\left(\tilde{x}_{i}^{(1)}\right)<\left(\tilde{x}_{i}^{(2)}\right)<\ldots<\left(\tilde{x}_{i}^{(n)}\right)<\ldots$ be a strictly increasing infinite sequence in $S P_{\omega}(X)$.

Define a function $R$ on $c_{00}$, the linear space of all finitely supported sequences of reals, by

$$
R(a)=\sup _{n}\left\|\sum_{i} a_{i} \tilde{x}_{i}^{(n)}\right\|
$$

for all $a=\left(a_{i}\right) \in c_{00}$. We will write $\left(\tilde{y}_{i}\right)<R$ if there exists a constant $K$ such that for all $a=\left(a_{i}\right) \in c_{00},\left\|\sum_{i} a_{i} \tilde{y}_{i}\right\| \leq K R(a)$, and for all $N \geq 1$ there exists $a=\left(a_{i}\right) \in c_{00}$ so that $R(a)>N\left\|\sum_{i} a_{i} \tilde{y}_{i}\right\|$. Then, clearly, we have $\left(\tilde{x}_{i}^{(n)}\right)<R$, for all $n$.

Let $C=\left\{\left(\tilde{y}_{i}\right) \in S P_{\omega}(X):\left(\tilde{y}_{i}\right)<R\right\}$. Let $\mathcal{P}$ be the class of all chains (totally ordered subsets) in $C$ which contain $\left(\tilde{x}_{i}^{(1)}\right)<\left(\tilde{x}_{i}^{(2)}\right)<\ldots<\left(\tilde{x}_{i}^{(n)}\right)<\ldots$. Partially order $\mathcal{P}$ by set inclusion, up to equivalence. That is, for all $A, B \in \mathcal{P}, A \subseteq B$ means for every spreading model in $A$ there is an equivalent spreading model in $B$. Since $\mathcal{P}$ is non-empty, by Zorn's lemma there exists a maximal chain $M \in \mathcal{P}$. We claim that the set $M$ satisfies the assumptions (i) and (ii) of Lemma 2.1 which in turn will yield the theorem.

First, we show that every countable chain $\left(\tilde{y}_{i}^{(n)}\right)_{i}$ in $C$ has an upper bound in $C$. Indeed, let $\left(\tilde{y}_{i}^{(n)}\right)_{i}$ be a chain in $C$. For all $n$, let $A_{n} \geq 1$ be the best constant satisfying $\left\|\sum_{i} a_{i} \tilde{y}_{i}^{(n)}\right\| \leq A_{n} R(a)$ for all $a=\left(a_{i}\right) \in c_{00}$. Let $\left(\tilde{z}_{i}\right)$ be a spreading model given by Theorem 1.1 applied to $\left(\tilde{y}_{i}^{(n)}\right)$ and $C_{n}=2^{n} A_{n}, n=1,2, \ldots$. Then $\left(\tilde{z}_{i}\right) \geq\left(\tilde{y}_{i}^{(n)}\right)$, for all $n$. That is, $\left(\tilde{z}_{i}\right)$ is an upper bound for $\left(\tilde{y}_{i}^{(n)}\right)_{i}$ in $S P_{\omega}(X)$. To see that $\left(\tilde{z}_{i}\right) \in C$, let $\varepsilon>0$ be arbitrary, and let $D$ be as in (1). Let $m \in \mathbb{N}$ such that $\sum_{n=m+1}^{\infty} 2^{-n}<\varepsilon / 2 D$. Since $\left(\tilde{y}_{i}^{(n)}\right)_{i}$ is a chain, there is $n_{0} \in\{1, \ldots, m\}$ such that $\left(\tilde{y}_{i}^{(n)}\right) \leq\left(\tilde{y}_{i}^{\left(n_{0}\right)}\right)$, for all $n \leq m$. Let $K_{0}=\max \left\{K_{1}, \ldots, K_{m}\right\}<\infty$, where each $K_{n}$ satisfies $\left\|\sum_{i} a_{i} \tilde{y}_{i}^{(n)}\right\| \leq K_{n}\left\|\sum_{i} a_{i} \tilde{y}_{i}^{\left(n_{0}\right)}\right\|$, for all $\left(a_{i}\right)$. By the fact that $\left(\tilde{y}_{i}^{\left(n_{0}\right)}\right)_{i} \nsucceq R$, we can pick $a_{\varepsilon}=\left(a_{i}^{\varepsilon}\right) \in c_{00}$ such that

$$
\frac{\left\|\sum_{i} a_{i}^{\varepsilon} \tilde{y}_{i}^{\left(n_{0}\right)}\right\|}{R\left(a_{\varepsilon}\right)}<\frac{\varepsilon}{2 D K_{0}}
$$


Then by (1)

$$
\begin{aligned}
\frac{\left\|\sum_{i} a_{i}^{\varepsilon} \tilde{z}_{i}\right\|}{R\left(a_{\varepsilon}\right)} & \leq D \sum_{n=1}^{m} 2^{-n} A_{n}^{-1} \frac{\left\|\sum_{i} a_{i}^{\varepsilon} \tilde{y}_{i}^{(n)}\right\|}{R\left(a_{\varepsilon}\right)}+D \sum_{n=m+1}^{\infty} 2^{-n} A_{n}^{-1} \frac{\left\|\sum_{i} a_{i}^{\varepsilon} \tilde{y}_{i}^{(n)}\right\|}{R\left(a_{\varepsilon}\right)} \\
& \leq D K_{0} \sum_{n=1}^{m} 2^{-n} \frac{\left\|\sum_{i} a_{i}^{\varepsilon} \tilde{y}_{i}^{\left(n_{0}\right)}\right\|}{R\left(a_{\varepsilon}\right)}+D \sum_{n=m+1}^{\infty} 2^{-n} \leq \varepsilon .
\end{aligned}
$$

Since $\varepsilon>0$ was arbitrary, this means that $\left(\tilde{z}_{i}\right) \geq R$. On the other hand, by (11) and (3) we have that $\left(\tilde{z}_{i}\right) \leq R$. Thus $\left(\tilde{z}_{i}\right)<R$, that is, $\left(\tilde{z}_{i}\right) \in C$.

To see that the set $M$ satisfies (ii) of Lemma 2.1, assume to the contrary that there exists a countable chain $\left(\tilde{y}_{i}^{(n)}\right) \subseteq M$ without an upper bound in $M$. Then by the maximality of $M$ in $C$, there is no such upper bound in $C$ either. But, since $\left(\tilde{y}_{i}^{(n)}\right)$ is a chain (as being a subset of $M$ ), by the above argument there is an upper bound $\left(\tilde{z}_{i}\right)$ in $C$, a contradiction.

To see (i), suppose that $M$ has a maximal element $\left(\tilde{u}_{i}\right)$. Then $\left(\tilde{u}_{i}\right)<R$. Thus for all $k \in \mathbb{N}$ there exists a sequence of scalars $a^{k}=\left(a_{i}^{k}\right)_{i=1}^{m(k)}$ such that

$$
\frac{\left\|\sum_{i=1}^{m(k)} a_{i}^{k} \tilde{u}_{i}\right\|}{R\left(a^{k}\right)}<\frac{1}{k 2^{k}} .
$$

For all $n$, let $\left(\tilde{y}_{i}^{(n)}\right) \in S P_{\omega}(X)$ be a spreading model obtained by Theorem 1.1 applied (merely) to two spreading models $\left(\tilde{u}_{i}\right)$ and $\left(\tilde{x}_{i}^{n}\right)$, and (coefficients) $C_{1}^{-1}=$ $C_{2}^{-1}=1 / 2$. Then $\left(\tilde{y}_{i}^{(n)}\right)$ 2-dominates $\left(\tilde{x}_{i}^{(n)}\right)$. That is, for all $\left(a_{i}\right), 2\left\|\sum_{i} a_{i} \tilde{y}_{i}^{(n)}\right\| \geq$ $\left\|\sum_{i} a_{i} \tilde{x}_{i}^{(n)}\right\|$. Observe that for all $n,\left(\tilde{y}_{i}^{(n)}\right)$ is equivalent to $\max \left\{\left(\tilde{u}_{i}\right),\left(\tilde{x}_{i}^{(n)}\right)\right\}$, which is, trivially, equivalent to $\left(\tilde{u}_{i}\right)$. In particular, they are all in $C$.

Now by (3), for all $k \in \mathbb{N}$ and $a^{k}=\left(a_{i}^{k}\right)_{i=1}^{m(k)}$ satisfying (4), there exists $n_{k} \in \mathbb{N}$ such that

$$
\left\|\sum_{i=1}^{m(k)} a_{i}^{k} \tilde{x}_{i}^{\left(n_{k}\right)}\right\| \geq \frac{1}{2} R\left(a^{k}\right) .
$$

In particular, for all $k$, we have

$$
\left\|\sum_{i=1}^{m(k)} a_{i}^{k} \tilde{y}_{i}^{\left(n_{k}\right)}\right\| \geq \frac{1}{4} R\left(a^{k}\right) .
$$

Now let $\left(\tilde{w}_{i}\right)$ be an upper bound obtained by Theorem 1.1 applied to $\left(\tilde{y}_{i}^{\left(n_{k}\right)}\right)$ and $\left(2^{-k}\right)$. Then again by the argument in the first part of the proof $\left(\operatorname{since}\left(\tilde{y}_{i}^{(n)}\right)\right.$ is a trivial chain, i.e., any two of them are mutually equivalent), we conclude that $\left(\tilde{w}_{i}\right)$ is in $C$, i.e., $\left(\tilde{w}_{i}\right)<R$. Also, clearly, $\left(\tilde{w}_{i}\right) \geq\left(\tilde{y}_{i}^{\left(n_{k}\right)}\right) \geq\left(\tilde{u}_{i}\right)$, for all $k$.

Moreover, for all $k \in \mathbb{N}$ we have

$$
\left\|\sum_{i=1}^{m(k)} a_{i}^{k} \tilde{w}_{i}\right\| \geq 2^{-k}\left\|\sum_{i=1}^{m(k)} a_{i}^{k} \tilde{y}_{i}^{\left(n_{k}\right)}\right\| \geq \frac{2^{-k}}{4} R\left(a^{k}\right) \geq \frac{k}{4}\left\|\sum_{i=1}^{m(k)} a_{i}^{k} \tilde{u}_{i}\right\| .
$$

This shows that $\left(\tilde{u}_{i}\right) \nsupseteq\left(\tilde{w}_{i}\right)$. Thus $\left(\tilde{u}_{i}\right)<\left(\tilde{w}_{i}\right)<R$. So $M$ is not a maximal chain, a contradiction. This completes the proof. 


\section{The UpPer EnVELOPE FunCtion $R_{X}$}

Define the upper envelope function $R_{X}^{\omega}$ for $S P_{\omega}(X)$ on $c_{00}$ as follows. For $a=$ $\left(a_{i}\right) \in c_{00}$, let

$$
R_{X}^{\omega}(a)=\sup \left\|\sum_{i} a_{i} \tilde{x}_{i}\right\|
$$

where sup is over all $\left(\tilde{x}_{i}\right)_{i} \in S P_{\omega}(X)$. It is easy to see that it defines a 1unconditional and 1-spreading norm on $c_{00}$. For $a=\left(a_{i}\right) \in c_{00}$, for convenience, we will sometimes write $R_{X}^{\omega}\left(\sum_{i} a_{i} e_{i}\right)$ for $R_{X}^{\omega}(a)$, where $\left(e_{i}\right)$ is the unit vector basis of $c_{00}$.

Then the following is proved easily using Theorem 1.1 (cf. Remark 3.5(a) of [AOST]). (Observe that Theorem 2.2 extends this proposition.)

Proposition 3.1. Suppose that $R_{X}^{\omega}$ is not equivalent to any spreading model in $S P_{\omega}(X)$. Then $S P_{\omega}(X)$ contains an uncountable increasing chain.

Let $\left(\tilde{x}_{i}\right) \in S P_{\omega}(X)$ and let $\left(\tilde{y}_{j}\right)$ be an identically distributed normalized block basis of $\left(\tilde{x}_{i}\right)$. That is, there exists $\alpha=\left(\alpha_{i}\right) \in c_{00}$ such that for all $j, \tilde{y}_{j}=\sum_{i \in \sigma_{j}} \alpha_{i} \tilde{x}_{i}$ with $\left\|\tilde{y}_{j}\right\|=1$ and $\sigma_{1}<\sigma_{2}<\ldots, \sigma_{i} \subset \mathbb{N}$. Then, clearly $\left(\tilde{y}_{j}\right) \in S P_{\omega}(X)$ as well. (Note, however, that in general a block basis of a spreading model is not necessarily a spreading model.) This property immediately implies the following sub-multiplicativity property for $R_{X}^{\omega}$.

Lemma 3.2 (纤). Let $\left(e_{i}\right)$ be the unit vector basis of $c_{00}$. Let $\left(u_{i}\right)$ be an identically distributed block basis of $\left(e_{i}\right)$ such that $R_{X}^{\omega}\left(u_{i}\right)=1$. Then for all $a=\left(a_{i}\right) \in c_{00}$, we have

$$
R_{X}^{\omega}\left(\sum_{i} a_{i} u_{i}\right) \leq R_{X}^{\omega}\left(\sum_{i} a_{i} e_{i}\right)
$$

Let $\left(\tilde{x}_{i}\right) \in S P_{\omega}(X)$. Then by Krivine's theorem there exists $1 \leq p \leq \infty$ such that for all $k \in \mathbb{N}$ and $\varepsilon>0$, there is an identically distributed block basis $\left(\tilde{y}_{i}\right)$ of $\left(\tilde{x}_{i}\right)$ so that any $k$ vectors of $\left(\tilde{y}_{i}\right)$ are $(1+\varepsilon)$-equivalent to the unit vector basis of $\ell_{p}^{k}$. The Krivine set of $\left(\tilde{x}_{i}\right)$ is the set of all $p$ 's satisfying the above assertion. Moreover, we define the Krivine set of a Banach space $X$ to be the union of the Krivine sets of all the spreading models of $X$.

The above lemma in turn implies, again by well-known standard arguments using Krivine's theorem (cf. Proposition 5.2, $\mathrm{Sa}$ ), that

Proposition 3.3. There exists $1 \leq p \leq \infty$ and for every $\varepsilon>0$ there exists $C_{\varepsilon}$ such that for all $a \in c_{00}$,

$$
\|a\|_{\ell_{p}} \leq R_{X}^{\omega}(a) \leq C_{\varepsilon}\|a\|_{\ell_{p-\varepsilon}} .
$$

Here it is understood that if $p=1$, then $R_{X}^{\omega}$ is equivalent to $\|\cdot\|_{\ell_{1}}$, and if $p=\infty$, then for all $r<\infty$ there exists $C_{r}$ such that $R_{X}^{\omega}(a) \leq C_{r}\|a\|_{\ell_{r}}$.

Observe that if 1 belongs to the Krivine set of a spreading model $\left(\tilde{x}_{i}\right) \in S P_{\omega}(X)$, then $R_{X}^{\omega}$ is equivalent to $\|.\|_{\ell_{1}}$. However, even in this case $R_{X}^{\omega}$ need not be equivalent to a spreading model in $S P_{\omega}(X)$ (the space with no $\ell_{p}$ spreading models constructed in $\mathrm{OS}$ is such an example). 
From Proposition 3.1 and Proposition 3.3 above we deduce the following, which gives a partial answer to Question 4.7 of [AOST.

Theorem 3.4. Let $X$ be a Banach space such that $S P_{\omega}(X)$ is countable. Then there exists $\left(\tilde{x}_{i}\right) \in S P_{\omega}(X)$ such that the following holds. There exist $1 \leq p \leq \infty$ and $c>0$, and for every $\varepsilon>0$ there exists $C_{\varepsilon}$ such that

$$
c\|a\|_{\ell_{p}} \leq\left\|\sum_{i} a_{i} \tilde{x}_{i}\right\| \leq C_{\varepsilon}\|a\|_{\ell_{p-\varepsilon}},
$$

for all $a=\left(a_{i}\right) \in c_{00}$. If $p=1$, then $\left(\tilde{x}_{i}\right)$ is equivalent to the unit vector basis of $\ell_{1}$. Moreover, the Krivine set of $\left(\tilde{x}_{i}\right)$ must be a singleton of $\{p\}$.

Following [FPR] we say that a normalized basis $\left(e_{i}\right)$ has Rosenthal's property or is a Rosenthal basis if every normalized block basis of $\left(e_{i}\right)$ has a subsequence that is equivalent to $\left(e_{i}\right)$. It is proved there that a Rosenthal basis is subsymmetric, that is, it is equivalent to its every subsequence and it is unconditional. A question due to $H$. Rosenthal asks whether such a basis is equivalent to the unit vector basis of $c_{0}$ or $\ell_{p}$, for some $1 \leq p<\infty$. If $X$ is non-reflexive, then by James' theorem $X$ contains a copy of either $c_{0}$ or $\ell_{1}$. This quickly implies that $\left(e_{i}\right)$ is equivalent to the unit vector basis of $c_{0}$ or $\ell_{1}$. So the non-trivial case is when $X$ is reflexive. For a reflexive $X$, clearly, every spreading model of $X$ generated by a block basis is equivalent to $\left(e_{i}\right)$. Thus, we have the following consequence of Theorem 3.4, which improves on the results in [FPR.

Corollary 3.5. Let $X$ be a reflexive Banach space with a Rosenthal basis $\left(e_{i}\right)$. Then there exists $1<p \leq \infty$ and for every $\varepsilon>0$ there exists $C_{\varepsilon}$ such that

$$
\|a\|_{\ell_{p}} \leq\left\|\sum_{i} a_{i} e_{i}\right\| \leq C_{\varepsilon}\|a\|_{\ell_{p-\varepsilon}}
$$

for all $a=\left(a_{i}\right) \in c_{00}$. If $p=\infty$, then for all $r<\infty$ there exists $C_{r}$ such that $\left\|\sum_{i} a_{i} e_{i}\right\| \leq C_{r}\|a\|_{\ell_{r}}$. Moreover, the Krivine set I of $X$ must be a singleton, i.e., $I=\{p\}$.

\section{ACKNOWLEDGMENTS}

The author thanks R. Anisca and T. Schlumprecht for helpful discussions, and especially E. Odell for many suggestions. He also thanks the anonymous referee for the invaluable suggestions which helped to improve the paper. The paper was written while the author held a visiting position at the University of South Carolina, and he thanks the Department of Mathematics and G. Androulakis for their hospitality.

\section{REFERENCES}

[AOST] G. Androulakis, E. Odell, Th. Schlumprecht and N. Tomczak-Jaegermann, On the structure of the spreading models of a Banach space, to appear in Canadian Journal of Math.

[BL] B. Beauzamy and J.-T. Lapreste, Modèles étalés des espaces de Banach, Travaux en Cours, Hermann, Paris, 1984. MR0770062 (86h:46024)

[FPR] V. Ferenczi, A.M. Pelczar and C. Rosendal, On a question of Haskell P. Rosenthal concerning a characterization of $c_{0}$ and $\ell_{p}$, Bull. London Math. Soc. 36 (2004), no. 3 , 396-406. MR2038727 (2004m:46026) 
[OS] E. Odell and T. Schlumprecht, On the richness of the set of $p$ 's in Krivine's theorem, Geometric aspects of functional analysis (Israel, 1992-1994), 177-198, Oper. Theory Adv. Appl., 77, Birkhaüser, Basel, 1995. MR1353459 (96i:46015)

[Sa] B. Sari, Envelope functions and asymptotic structures in Banach spaces, Studia Math. 164 (2004), no. 3, 283-306. MR2079954

Department of Mathematics, University of South Carolina, Columbia, South CarOLINA 29208

E-mail address: bsari@math.sc.edu 